\title{
Translating Value-Based Purchasing into Value-Based Care
}

Yasir S Alsalamah ${ }^{1 *}$

${ }^{1}$ MSN, RN, Department of Nursing, Critical Care, King Abdulaziz Medical City, Ministry of National Guard-Health Affairs, POB 22490, Riyadh, 11426, Saudi Arabia

DOI: $10.36348 /$ sjnhc.2021.v04i03.007 $\quad$ | Received: 05.03.2021 | Accepted: 24.03.2021 | Published: 28.03 .2021

*Corresponding author: Yasir S Alsalamah

\section{Abstract}

High quality, collaborative and patient-centered care is essential in the modern healthcare industry. The Hospital ValueBased Purchasing (VBP) Program is a CMS initiative that rewards acute-care hospitals with incentive payments for the quality of care they provide to people. It is expected for the on-deck years that VBC will be practical and effective and help provide the organizational incentive necessary to recognize a need for change in the way healthcare business is currently conducted. There are several healthcare settings, but not all provide patient-centric care facilities. The valuebased care model invests many of its resources on the nursing staff, thereby creating a home-like ambiance for the patients. Nurses remain in direct communication with the patients at all times and monitor their health trends thoroughly. This develops a relationship of trust between the nursing staff and the patient. When nurses are welcoming and polite, the patients begin to feel that they are being cared for, which helps them recover faster.

Keywords: patient-centered care, modern healthcare industry, CMS initiative.

Copyright () 2021 The Author(s): This is an open-access article distributed under the terms of the Creative Commons Attribution 4.0 International License (CC BY-NC 4.0) which permits unrestricted use, distribution, and reproduction in any medium for non-commercial use provided the original author and source are credited.

\section{Value Based Purchasing Performance Metrics}

Hackensack UMC is a general hospital and nursing home offering healthcare services to thousands of people across New Jersey. The hospital provides services to diagnose and treat diseases alongside obstetrical care to the ones in need [1]. At Hackensack UMC, long-term care facilities are also available, including nursing, therapy, and diagnostic testing.

This setting's organizational structure enables convenient monitoring and control of activities and simplifies tasks by disintegrating processes into fractions. Closest to the patient lie the doctors, nurses, paramedical staff, and the quality control department. These are directly headed by a medical superintendent whom a director leads. The director also heads the general manager, finance manager, marketing manager, and purchase manager, under whose supervision, work for the accounts personnel, HR department, PRO, and store managers. Since Hackensack UMC adheres to the Six Sigma Principles, teams are often made with respect to particular projects which a project manager leads [1].

The structure invests capital in training the nursing staff, offering them incentives and accolades to form a strong link between quality care services and employee satisfaction. Nurses being the closest to a patient offering first-hand care can influence the overall system a great deal. Through empirical outcomes, it has been demonstrated that with outstanding clinical nursing practice and a healthy work environment, a value-based care infrastructure is bred, taking into account safety, efficiency, effectiveness, patientcenteredness, and time.

The Hospital Value-Based Purchasing Program (HVBPP) is one of the many quality indicators used to determine care quality. It covers eight dimensions and generates a collective score to weigh the hospitals' performance. These include communication with nurses, communication with doctors, staff responsiveness, pain management, communication about medicines, discharge information, cleanliness and quietness of the hospital, and overall rating. The overall score is often calculated by multiplying the sum of each domain's performance scores by the weight of that domain [1]. VBP translates to performance reward reimbursement for accountability and excellence in health care by providing better value and better patient outcomes measured quantitatively through improvement data in the domains of the clinical process of care and patient experience of care. Clinical Process of Care Domain Score accounts for $70 \%$ of the VBP total score [2]. 
Creating an environment where the patients feel safe and sound is something all healthcare units strive for. The first thing is to ensure that the people in charge, such as the lead medical physicians, the directors, the chief nursing member, etc., all attempt to develop a safe culture within the unit and outside [2]. Centralized and well-coordinated oversight of patient safety comes next. It is always better to delineate safety metrics beforehand for quick evaluation. Also, increasing the funding for patient safety research with programs like High-Quality Incentive Demonstration (HQID) and Physician Group Practice (PGP) create much difference. Following this comes the implementation of safety programs throughout the care unit and extending to the workforce. The technology being used should also be checked thoroughly at regular intervals to meliorate patient safety.
Several changes like creating awareness amongst people about patient safety, improving reward reimbursement programs, and training the nursing and paramedic staff to be compassionate and empathetic, need to be made to enhance the already-established culture of safety further.

\section{REFERENCES}

1. Aroh, D., Colella, J., \& Douglas, C. (March-April 2015). An Example of Translating Value-Based Purchasing Into Value-Based Care. Urologic Nursing. DOI:10.7257/1053-816X.2015.35.2.61

2. Fallon, L. F., Begun, J. W., \& Riley, W. (2013). Managing health organizations for quality and performance. Burlington, MA: Jones \& Bartlett. ISBN 987-1-4496-5327-9. 